\title{
NOTE ON A THEOREM OF NEHARI ON HANKEL FORMS
}

\author{
JU-KWEI WANG
}

Let $\Gamma$ be a discrete ordered abelian group and let $G$ be its Pontrjagin dual, both written in additive notation. It is known [3, Chapter 8] that $G$ is compact and connected. We denote the set of nonnegative elements of $\Gamma$ by $\Gamma^{+}$. Then a Hankel form on $\Gamma$ is defined to be a complex bilinear form

$$
A(a, b)=\sum_{\mu, \nu \in \Gamma^{+}} \phi(\mu+\nu) a(\mu) b(\nu),
$$

where $a, b$ and $\phi$ are functions on $\Gamma^{+}$. If

$$
|A(a, b)| \leqq M<\infty
$$

for all functions $a$ and $b$ with

$$
\sum_{\mu \in \Gamma^{+}}|a(\mu)|^{2}=\sum_{\nu \in \Gamma^{+}}|b(\nu)|^{2}=1,
$$

then the Hankel form $A$ is called bounded and $M$ is called a bound of $A$. The purpose of this note is to prove the following criterion of boundedness of Hankel forms:

TheOREM. The Hankel form (1) on $\Gamma$ is bounded with a bound $M$ if and only if there is a function $f \in L^{\infty}(G)$ with respect to the normalized Haar measure $d x$ on $G$ such that

$$
\|f\|_{\infty} \leqq M
$$

and

$$
\phi(\mu)=\int f(x)(x, \mu) d x, \quad \mu \in \Gamma^{+} .
$$

In particular this theorem implies that the least bound of $A$ may be realized by the $L^{\infty}$-norm of some $f$ satisfying these conditions. This is exactly where the crux of the proof lies.

This theorem is due to Nehari [2] in case $\Gamma$ is the additive group of integers and $G$ is the circle group. His proof relies heavily on complex analysis and does not generalize to our case. Our technique is drawn from the theory of Hardy classes [1], [3].

Received by the editors March 28, 1969. 
The sufficiency of the conditions (3) and (4) is immediate. In fact let $a, b$ be given satisfying (2). Then by Plancherel's theorem, the functions $\alpha, \beta$ in the Hardy class $H^{2}(G)$ defined by the mean-convergent series

$$
\alpha(x)=\sum_{\mu \in \Gamma^{+}} a(\mu)(x, \mu), \quad \beta(x)=\sum_{\nu \in \Gamma^{+}} b(\nu)(x, \nu)
$$

have $L^{2}$-norms equal to 1 . Further

$$
\begin{aligned}
|A(a, b)| & =\left|\sum_{\mu, \nu \in \Gamma^{+}} \int f(x)(x, \mu+\nu) a(\mu) b(\nu) d x\right| \\
& =\left|\int f(x) \alpha(x) \beta(x) d x\right| \leqq\|f\|_{\infty}\|\alpha\|_{2}\|\beta\|_{2} \leqq M .
\end{aligned}
$$

Next we like to prove the necessity part of the theorem. Thus let $A$ be a Hankel form on $\Gamma$ bounded by $M$. For a function $g \in H^{1}(G)$, let the Fourier coefficients of $g$ be

$$
c(\mu)=\int g(x) \overline{(x, \mu)} d x, \quad \mu \in \Gamma^{+} .
$$

Then we claim that the series

$$
\sum_{\mu \in \Gamma^{+}} \phi(\mu) c(\mu)
$$

converges. In fact, let $h$ and $k$ be the inner and outer functions such that $g=h k$. Put $\alpha=h k^{1 / 2}, \beta=k^{1 / 2}$, the square root being taken in a continuous fashion. Then from the theory of Dirichlet algebras [1], $\alpha, \beta \in H^{2}$, and $\|\alpha\|_{2}=\|\beta\|_{2}=\|g\|_{1}^{1 / 2}$. Denote the Fourier coefficients of $\alpha$ and $\beta$ by $a(\mu)$ and $b(\nu)$ respectively. Then

$$
\sum|a(\mu)|^{2}=\sum|b(\nu)|^{2}=\|g\|_{1} ;
$$

and (5) is reduced to $A(a, b)$, and is therefore convergent. Define the sum of (5) as $T g$. $T$ is clearly a linear functional on $H^{1}$. Since

$$
|T g|=|A(a, b)| \leqq M\|a\|_{2}\|b\|_{2}=M\|g\|_{1},
$$

$T$ is a bounded linear functional on $H^{1}$ with norm not exceeding $M$. By the Hahn-Banach theorem there is a norm-preserving extension of $T$ to $L^{1}(G)$ which is given by some $L^{\infty}$ function $f$ :

$$
T g=\int f(x) g(x) d x, \quad g \in L^{1},
$$


satisfying (3). The condition (4) is then a consequence of (6) by taking $g(x)=(x, \mu)$. The theorem is therewith proved.

\section{REFERENCES}

1. K. Hoffman, Banach spaces of analytic functions, Prentice-Hall Series in Modern Analysis, Prentice-Hall, Englewood Cliffs, N. J., 1962. MR 24 \#A2844.

2. Z. Nehari, On bounded bilinear forms, Ann. of Math. (2) 65 (1957), 153-162. MR 18, 633.

3. W. Rudin, Fourier analysis on groups, Interscience Tracts in Pure and Appl. Math., no. 12, Interscience, New York, 1962. MR 27 \#2808.

University of Massachusetts 